\title{
Nutrição mineral do mangarito num Latossolo Vermelho Amarelo
}

\author{
Candido A da Costa; Sílvio Junio Ramos; Daniel S Alves; Luiz Arnaldo Fernandes; Regynaldo A \\ Sampaio; Ernane Ronie Martins \\ UFMG - NCA, C. Postal 135, 39404-006 Montes Claros-MG; larnaldo@ufmg.br
}

\begin{abstract}
RESUMO
Com o objetivo de caracterizar as limitações nutricionais para a produção de matéria seca do mangarito [(Xanthosoma mafaffa (L.) Schott)], conduziu-se um experimento em casa de vegetação com amostras da camada de 0-20 cm de um Latossolo Vermelho Amarelo sob vegetação de cerrado, coletado no município de Montes Claros, MG. O cultivo foi realizado em vasos de $3 \mathrm{~L}$ de capacidade. $\mathrm{O}$ delineamento experimental foi inteiramente casualizado, em treze tratamentos, com três repetições. Os tratamentos foram baseados na técnica do elemento faltante (em que se omite a calagem e cada um dos macronutrientes e micronutrientes). A ordem decrescente dos tratamentos que mais afetaram a produção de matéria seca da parte aérea foi: -Calagem $>-\mathrm{P}>-\mathrm{S}>$ testemunha $>-\mathrm{B}>-$-Calagem $+\mathrm{Ca}+\mathrm{Mg}$ $>-\mathrm{N}>$-K. A omissão de Ca, Mg, Zn e Cu não afetaram a produção de matéria seca da parte aérea. A ordem decrescente dos tratamentos que mais afetaram a produção de matéria seca de raízes foi: -B > $\mathrm{Mg}>$-Calagem+Ca+Mg $>-\mathrm{K}>-\mathrm{N}>-\mathrm{Zn}>$-Calagem $>-\mathrm{S}>-\mathrm{Cu}>$ $-\mathrm{Ca}>-\mathrm{Cu}>-\mathrm{Ca}>$ testemunha $>-\mathrm{P}$.
\end{abstract}

Palavras-chave: Xanthosoma mafaffa, requerimento de nutrientes, hortaliça não convencional.

\begin{abstract}
Mineral nutrition of Xanthosoma mafaffa under cerrado soil

The aim of this work was to characterize the nutritional limitations for dry matter production of Xanthosoma mafaffa (L.) Schott. The experiment was carried out in a greenhouse with 0-20 cm samples of a Oxisol under cerrado vegetation, collected at Montes Claros, Minas Gerais State, Brazil. The cultivation was conducted in $3 \mathrm{~L}$ pots. The experimental design was totally randomized with 13 treatments and three replications. Treatments were based on the missing element technique (with omission of liming and each one of the macro and micronutrients). The decreasing order of treatments that more influenced the aerial dry matter production was: -liming> $-\mathrm{P}>-\mathrm{S}>$ test $>-\mathrm{B}>-$ liming $+\mathrm{Ca}+\mathrm{Mg}>-\mathrm{N}>-\mathrm{K}$. The omission of $\mathrm{Ca}, \mathrm{Mg}, \mathrm{Zn}$ and $\mathrm{Cu}$ did not influence the aerial dry matter production. The decreasing order of treatments that influenced the root dry matter production was: $-\mathrm{B}>-\mathrm{Mg}>-$ liming $+\mathrm{Ca}+\mathrm{Mg}>-\mathrm{K}>-\mathrm{N}>-\mathrm{Zn}>-$ liming $>-\mathrm{S}>-\mathrm{Cu}>-\mathrm{Ca}>-\mathrm{Cu}>-\mathrm{Ca}>$ test $>-\mathrm{P}$.
\end{abstract}

Keywords: Xanthosoma mafaffa, nutrients requirement, no conventional vegetable crops.

\section{(Recebido para publicação em 19 de novembro de 2006; aceito em 18 de fevereiro de 2008)}

A crescente expansão demográfica e suas possíveis conseqüências, em termos de demanda de alimentos, tem incentivado os pesquisadores a procurar novas opções, dentre as fontes já utilizadas e atualmente esquecidas, ou a fomentar o uso daquelas pouco conhecidas (Klein et al.,1989).

Existe hoje um crescente interesse do consumidor por novidades na área alimentar, o que influencia também o mercado de hortaliças (Junqueira e Luengo, 2000).

O gênero Xanthosoma, da família Araceae, inclui muitas espécies, como o mangarito [(Xanthosoma mafaffa (L.) Schott)] que é uma das mais difundidas e cultivadas (Coursey, 1968; Pena, 1970). O mangarito é originário da região centro-americana, e é encontrado no México, Venezuela, Colômbia, Panamá, Costa Rica, Peru e Brasil. É também chamado de tannia, tiquisque, malangay e no Brasil, é também conhecido como mangará, taioba portuguesa e mangareto.
A espécie apresenta rizoma subterrâneo principal, com brotações laterais e várias folhas grandes brotam do rizoma principal. As inflorescências são raramente férteis, produzindo poucas sementes viáveis. Apresenta ciclo em torno de 11 meses sendo que durante os primeiros seis meses ocorre o desenvolvimento do rizoma e folhas e nos últimos quatro meses as folhas começam a secar, indicando o ponto de colheita da cultura.

Pelas características culinárias peculiares de seus rizomas, são muito apreciados pela população rural e só é comercializado sazonalmente, em locais próximos às áreas de produção. Todavia, progressos tecnológicos que levem ao aumento da produção e da qualidade comercial dos rizomas, poderão tornálo um produto mais popular para o mercado horti-granjeiro nacional.

Os rizomas são consumidos cozidos, ensopados com carnes e ao molho e seu valor nutricional é comparável à batata. Um uso secundário está no consumo das folhas jovens, que são comparadas ao espinafre. Em Porto Rico, pelo processo de desidratação, é consumido também na forma de farinha. Segundo Jordan (1979), as características nutritivas de mangarito são: $9 \mathrm{~g}$ de calorias; 70,5 g de água; 2,5 g de proteínas; 27 g de carboidratos; $16 \mathrm{mg}$ de cálcio; $47 \mathrm{mg}$ de fósforo; 0,9 $\mathrm{mg}$ de ferro e $7 \mathrm{mg}$ de vitamina C. Devido às características de valor nutricional, o mangarito pode ser sugerido juntamente com outras espécies produtoras de tubérculos e raízes tuberosas, como cultura alternativa para aumentar a base alimentar de regiões em desenvolvimento.

Os dados técnicos sobre seu cultivo são escassos e, para sua propagação e plantio podem ser usados tanto os rizomas primários como os secundários (Eny, 1967). No entanto, os estudos realizados por Vasconcelos (1972) estabeleceram algumas bases racionais para a cultura, como a profundidade de plantio, o espaçamento, o tipo e o tamanho de rizomas-semente. 
O estresse nutricional decorrente da deficiência ou do excesso de nutrientes ou de elementos químicos que interferem no metabolismo vegetal é um importante fator na redução da produtividade, notadamente em solos tropicais de baixa disponibilidade de nutrientes e elevada acidez, como os do Norte de Minas Gerais onde o mangarito é cultivado por pequenos agricultores de forma rudimentar, sem aplicação de fertilizantes.

A eficiência nutricional, do ponto de vista fisiológico, refere-se à habilidade de uma planta em absorver o nutriente do solo, distribuí-lo e utilizá-lo internamente (Goddard \& Hollis, 1984). Para Graham, (1984), a eficiência de uso de um nutriente é definida como a habilidade de uma espécie ou genótipo em fornecer altas produções num solo deficiente no nutriente em estudo. Para o mangarito, não foi encontrada na literatura consultada a descrição de sintomas de deficiências de nutrientes.

Embora seja tradicionalmente cultivada no norte de Minas Gerais, poucas informações existem a respeito desta espécie, principalmente as relativas ao manejo e as exigências nutricionais da cultura. Nessa região as plantas são cultivadas por pequenos agricultores, preferencialmente nas áreas baixas da paisagem, próximas aos cursos d’água, onde os solos apresentam teores mais elevados de matéria orgânica e de nutrientes. Com a retirada da vegetação nativa e cultivo intensivo dessas áreas a produção de mangarito está decrescendo a cada cultivo, reduzindo a renda das famílias. Desta forma o objetivo deste trabalho foi caracterizar as limitações nutricionais no crescimento do mangarito [(Xanthosoma mafaffa (L.) Schott)], utilizando a técnica do elemento faltante.

\section{MATERIAL E MÉTODOS}

O experimento foi conduzido em casa de vegetação, na UFMG - NCA, em Montes Claros, de novembro de 2004 a julho de 2005. Utilizaram-se amostras $(0-20 \mathrm{~cm})$ de solo Latossolo Vermelho Amarelo distrófico e álico, coletado no município de Montes Claros. Após a coleta o solo foi peneirado em malha de $5 \mathrm{~mm}$ e determinados os atributos físicos e químicos, conforme Embrapa (1997): pH em água= 4,6; $\mathrm{P}=$ $0,6 \mathrm{mg} \mathrm{dm}^{-3}, \mathrm{~K}=0,01 \mathrm{mmol}_{\mathrm{c}} \mathrm{dm}^{-3} ; \mathrm{Ca}=$ $1,1 \mathrm{mmol}_{\mathrm{C}} \mathrm{dm}^{-3} ; \mathrm{Mg}=0,4 \mathrm{mmol}_{\mathrm{C}} \mathrm{dm}^{-3}$; $\mathrm{Al}=37 \mathrm{mmol}_{\mathrm{c}} \mathrm{dm}^{-3} ; \mathrm{H}+\mathrm{Al}=120 \mathrm{mmol}_{c}$ $\mathrm{dm}^{-3} ; \mathrm{SB}=1,51 \mathrm{mmol}_{\mathrm{c}} \mathrm{dm}^{-3} ; \mathrm{t}=38,51$ mmol $\mathrm{dm}^{-3}$; Matéria orgânica $=24 \mathrm{~g} \mathrm{~kg}^{-1}$; areia $=500 \mathrm{~g} \mathrm{~kg}^{-1}$, silte $=80 \mathrm{~g} \mathrm{~kg}^{-1}$ e argi$\mathrm{la}=420 \mathrm{~g} \mathrm{~kg}^{-1}$.

Foi realizado o cultivo do mangarito, em vasos de $3 \mathrm{~L}$ de solo. O delineamento experimental foi o inteiramente casualizado, sendo 13 tratamentos, com três repetições. Os tratamentos, baseados na técnica do elemento faltante foram: 1) testemunha (T); 2) completo 1 : adubado com N, P, K, S, B, Cu e Zn + calagem (C1); 3) completo 2: completo 1 -calagem + Ca e Mg como sulfato (C2); 4) Completo 1 -calagem (C1calagem); 5) completo $1-\mathrm{N}(\mathrm{C} 1-\mathrm{N}) ; 6)$ completo 1 -P (C1-P); 7) completo 1 $\mathrm{K}$ (C1-K); 8) completo 1 -S (C1-S); 9) completo 1 -B (C1-B); 10) completo 1 -Cu (C1-Cu); 11) completo 1 -Zn (C1$\mathrm{Zn})$; 12) completo 2 -Ca (C2-Ca) e 13) completo 2 -Mg (C2-Mg).

Nos tratamentos que receberam calcário, as doses foram baseadas em curvas de incubação obtidas em experimentos realizados em laboratório, visando elevar a saturação por bases (V) a $70 \%$. O corretivo utilizado foi o calcário dolomítico calcinado e micropulverizado, com 36\% de $\mathrm{CaO}$, $14 \%$ de $\mathrm{MgO}$, PRNT igual a $100 \%$, 0,02\% de Zn, 0,01\% de B, 0,03\% de $\mathrm{Fe}, 0,03 \%$ de $\mathrm{Mn}$ e $0,00 \%$ de $\mathrm{Cu}$.

Para o cultivo, foram aplicados por $\mathrm{dm}^{3}$ de solo, $100 \mathrm{mg}$ de $\mathrm{N}, 300 \mathrm{mg}$ de $\mathrm{P}_{2} \mathrm{O}_{5}, 100$ mg de $\mathrm{K}_{2} \mathrm{O}$, $200 \mathrm{mg}$ de $\mathrm{Ca}, 60$ mg de Mg, 40 mg de S, 0,5 mg de B, 1,5 mg de $\mathrm{Cu}$ e 5 mg de $\mathrm{Zn}$, omitindo-se quando pertinente o nutriente referente ao tratamento. As doses das fontes foram calculadas de modo a atender corretamente à adubação básica para cada tratamento, de acordo com Malavolta (1980).

Após a aplicação do calcário nos tratamentos pertinentes, todos os vasos do experimento permaneceram em incubação por 30 dias, com umidade correspondente capacidade de campo. No final desses 30 dias, os solos foram seco e receberam os tratamentos, permane- cendo incubados por mais 30 dias, com umidade correspondente a capacidade de campo. Posteriormente, foi plantado um rizoma de 55 g em média de mangarito por vaso. A umidade dos vasos foi mantida em torno de $70 \%$ do volume total de poros, aferida mediante pesagens periódicas dos vasos, completando-se o peso com água desmineralizada.

Após a secagem do material vegetal, em estufa a $65-70^{\circ} \mathrm{C}$, foi determinada a matéria seca da parte aérea (hastes+folhas) (MSPA) e sistema radicular (rizomas+pequenas raízes) (MSSR) das plantas colhidas.

As variáveis foram submetidas à análise de variância e os tratamentos comparados pelo teste de Scott Knott a 5\% de probabilidade. Foi calculada, também, a produção relativa de matéria seca do sistema radicular e de matéria seca da parte aérea dos tratamentos de omissão em relação ao tratamento Completo 1 (100\%).

\section{RESULTADOS E DISCUSSÃO}

Os tratamentos estudados influenciaram tanto a produção de matéria seca do sistema radicular (MSSR) quanto a produção de matéria seca da parte aérea (MSPA). O tratamento C1 superou os demais tratamentos quanto a MSSR (Figura 1). A produção relativa de MSSR em todos os tratamentos estudados foi inferior a 50\% daquela obtida no tratamento C1 (Figura 2).

O tratamento C2 e os tratamentos com omissão de calagem C1- Cal, ou N (C1-N), ou K (C1-K), ou Mg (C1-Mg), ou Zn (C1-Zn), ou B (C1-B) foram os que mais limitaram o crescimento e o desenvolvimento do sistema radicular, reduzindo a MSSR a valores inferiores a $40 \%$ em relação ao tratamento completo 1 (Figura 2). Chaminade (1972) considera que a deficiência de um nutriente no solo é severa quando o rendimento da cultura é reduzido a valores inferiores a $40 \%$ em relação ao tratamento completo.

Quanto à produção de matéria seca da parte aérea (MSPA) verificou-se que não houve diferença significativa entre os tratamentos C1, C2-Ca, C2-Mg, C1$\mathrm{Zn}$ e C1-Cu. Nesses tratamentos a 


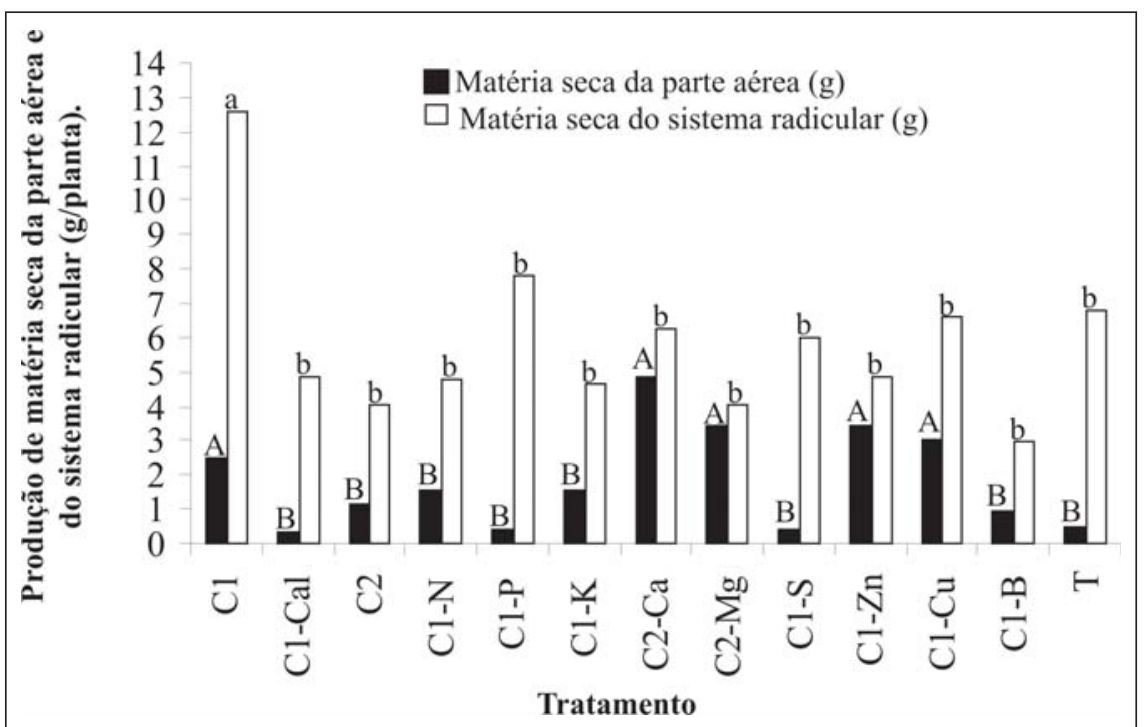

Figura 1. Produção de matéria seca da parte aérea e do sistema radicular de mangarito, em função dos tratamentos: C1= N, P, K, S, B, Cu e Zn + calagem; C1-Cal= C1 - calagem; C2= $\mathrm{C} 1$ - calagem + Ca e Mg como sulfato; C1-N= C1 - nitrogênio; C1-P= C1 - fósforo; C1-K= $\mathrm{C} 1$ - potássio; C2-Ca= C2 - cálcio; $\mathrm{C} 2-\mathrm{Mg}=\mathrm{C} 2$ - magnésio; $\mathrm{C} 1-\mathrm{S}=\mathrm{C} 1-$ enxofre; $\mathrm{C} 1-\mathrm{Zn}=$ $\mathrm{C} 1$ - zinco; $\mathrm{C} 1-\mathrm{Cu}=\mathrm{C} 1$ - cobre; $\mathrm{C} 1-\mathrm{B}=\mathrm{C} 1$ - boro e $\mathrm{T}=$ testemunha. Montes Claros, UFMGNCA, 2005.

*Médias seguidas pela mesma letra, maiúscula para matéria seca da parte aérea e minúscula para matéria seca do sistema radicular, não diferem entre si pelo teste de Scott Knott (1\% p).

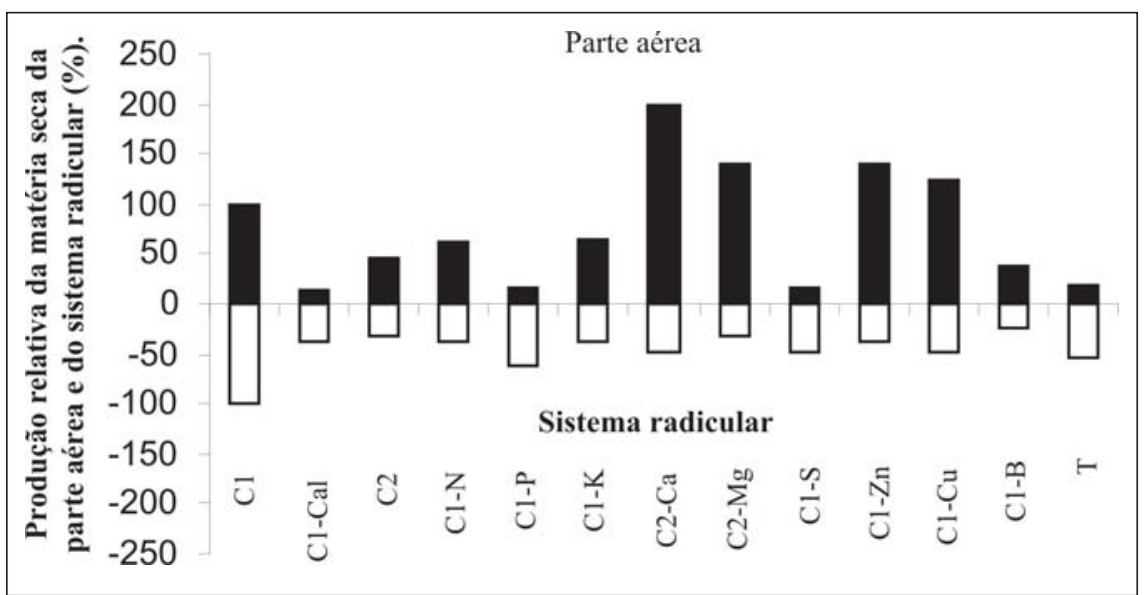

Figura 2. Produção relativa de matéria seca da parte aérea e do sistema radicular de mangarito em comparação ao tratamento C1. C1= N, P, K, S, B, Cu e Zn + calagem; C1-Cal= C1 calagem; $\mathrm{C} 2=\mathrm{C} 1$ - calagem + Ca e Mg como sulfato; $\mathrm{C} 1-\mathrm{N}=\mathrm{C} 1$ - nitrogênio; $\mathrm{C} 1-\mathrm{P}=\mathrm{C} 1$ fósforo; $\mathrm{C} 1-\mathrm{K}=\mathrm{C} 1$ - potássio; $\mathrm{C} 2-\mathrm{Ca}=\mathrm{C} 2$ - cálcio; $\mathrm{C} 2-\mathrm{Mg}=\mathrm{C} 2-$ magnésio; $\mathrm{C} 1-\mathrm{S}=\mathrm{C} 1$ enxofre; $\mathrm{C} 1-\mathrm{Zn}=\mathrm{C} 1$ - zinco; $\mathrm{C} 1-\mathrm{Cu}=\mathrm{C} 1$ - cobre; $\mathrm{C} 1-\mathrm{B}=\mathrm{C} 1$ - boro e $\mathrm{T}=$ testemunha. Montes Claros, UFMG, 2005.

MSPA foi significativamente superior à obtida nos demais. Os tratamentos que mais influenciaram foram C1-Cal, C1P e C1-S.

A omissão da calagem (C1-Cal), reduziu significativamente a MSPA e MSSR e corresponderam $12,6 \%$ e a $38,68 \%$, respectivamente, em relação ao tratamento C1, indicando que a ausência de calagem é um fator limitante para o crescimento do mangarito. Nessas condições a falta de calagem no solo contribuiu para um baixo valor de $\mathrm{pH}$, $\mathrm{Ca}$ e $\mathrm{Mg}$, e os nutrientes que compunham esse tratamento não puderam assumir a forma disponível para a planta (Marschner, 1995). Sob condições muito ácidas ocorre considerável solubilização do alumínio do solo o qual passa a ser um componente da acidez potencial e, consequentemente, por ser um elemento fitotóxico, um dos responsáveis pelos efeitos desfavoráveis da acidez sobre os vegetais.

Em geral, os efeitos perniciosos do alumínio nas plantas refletem-se principalmente nas raízes. Segundo Aguiar (1998), a toxidez por $\mathrm{Al}^{+3}$ reduz a divisão celular, impede a produção de reguladores de crescimento nas partes novas das raízes, impossibilitando a divisão celular e a absorção de $\mathrm{Ca}^{+2}$, que só ocorre na região apical da raiz. Assim, as raízes tornam-se mais lentas em alongar, engrossando e não se ramificando normalmente. Em solos com elevados teores de alumínio a calagem é imprescindível para o bom rendimento das culturas, pois além de reduzir ou eliminar os efeitos tóxicos do alumínio, aumenta a absorção de fósforo pelas plantas (Fernandes et al., 2000).

Por outro lado, algumas plantas nativas do Cerrado brasileiro são tolerantes ao alumínio tóxico, não respondo à calagem (Haridasan, 2000).

Quanto aos macronutrientes, os tratamentos C1-P e C1-S foram os mais limitantes para a MSPA (Figura 1). Quando se omitiu o P, a produção de matéria seca da parte aérea correspondeu a $15,44 \%$ e do sistema radicular a $61,95 \%$, em relação ao tratamento C1 (Figura 2). Segundo Lopes (1983), o P é o elemento que mais limita a produção das culturas, principalmente quanto se trata de solos do cerrado. Provavelmente a deficiência de $\mathrm{P}$ não permitiu uma multiplicação celular adequada para o crescimento da planta.

De acordo com Mengel \& Kirkby (1987), plantas com deficiência em fósforo têm o seu crescimento prejudicado, devido ao $\mathrm{P}$ participar de compostos que armazenam e fornecem energia para os diversos processos metabólicos da planta, tais como respiração, fotossíntese, síntese e desdobramento de carboidratos, trabalho mecânico e absorção iônica ativa. De acordo com Gomes \& Paiva, (2004) o fósforo é necessário para as plantas em quantidades bem menores do que as de nitrogênio e de potássio e, que um adequado suprimento desse elemento é importante no início do crescimento da planta para a formação dos primórdios vegetativos. 
Na omissão de S, a MSPA e a MSSR corresponderam, respectivamente, a $17,47 \%$ e a $47,81 \%$ da produção do tratamento C1 (Figura 2). Segundo Marschner (1995), o enxofre é um dos nutrientes básicos para a síntese de proteínas, e o suprimento inadequado desse nutriente acarreta em um menor desenvolvimento da parte aérea. De acordo com Carneiro (1995), o enxofre é essencial para uma eficiente utilização de $\mathrm{N}$ pelas plantas por ser constituinte de aminoácidos e essencial para a síntese de gorduras e, na sua ausência, as raízes se tornam mais fibrosas, compridas, mas com poucas radículas.

A omissão de $\mathrm{N}$ e de K também afetaram a produção de biomassa (Figura 1). No tratamento C1-N a MSPA correspondeu a $63 \%$ e a MSSR correspondeu a 38,12\% em comparação ao tratamento C1. De acordo com Carneiro (1995), esse elemento é importante na composição de clorofila, enzimas, proteínas estruturais, ácidos nucléicos e outros compostos orgânicos. Um bom suprimento de N para as plantas, segundo Scheller (1998), promove desenvolvimento maior nas folhas, o período vegetativo se torna mais longo e o teor de clorofila nas folhas aumenta, assim como a assimilação e a troca de energia e substâncias dentro da rizosfera.

Taiz \& Zeiger (2004) enfatizam que o N é o nutriente que mais altera a composição química na planta. Sob alta disponibilidade de $\mathrm{N}$, os teores de proteínas e aminoácidos solúveis na folha aumentam e o teor de açúcares diminui, além de retardar a maturação dos tecidos e diminuir sua lignificação. No entanto, a adubação nitrogenada em excesso pode provocar carência de cobre, ferro, manganês e zinco (Gomes, 2000).

No tratamento C1-K a produção de MSPA e MSSR diferiu estatisticamente em relação ao tratamento C1 (Figura 1) e corresponderam a 63,41\% e 36,85\%, respectivamente, em comparação ao tratamento C1. De acordo com Raij (1990), para um crescimento vigoroso e saudável, as plantas necessitam absorver grande quantidade de potássio. Esse elemento, segundo o autor, está envolvido na maioria dos processos biológicos da planta, atuando no suco celular. Segundo Araújo (2001), o K atua como ativador enzimático de processos responsáveis pela síntese e degradação de compostos orgânicos, participa no processo de abertura e fechamento dos estômatos, respiração, síntese de proteínas, osmorregulação, extensão celular e balanço entre cátions e anions. É indispensável também para a formação de proteínas, ou seja, o aproveitamento do nitrogênio.

Gomes (2000) afirma que com a deficiência de $K$, as plantas não conseguem usar a água do solo, prejudicando a absorção dos demais nutrientes, no entanto, a absorção excessiva de potássio causa prejuízo na absorção de zinco e de cálcio, o que aumento o efeito tóxico do manganês, em algumas situações.

Por outro lado, a omissão de Ca e Mg não afetaram a MSPA (Figura 1), pois não houve diferença significativa entre os tratamentos C1 e C2-Ca. No entanto, na ausência desses elementos a MSSR foi prejudicada em relação ao tratamento C1. O cálcio é imprescindível para a absorção dos nutrientes, manutenção da estabilidade das membranas e das paredes celulares das raízes e elongação e divisão celular, estando também envolvido no metabolismo do N (Marschner, 1995). Já a deficiência de Mg afeta a absorção de nutrientes (Malavolta, 1989).

Em relação aos tratamentos com omissão dos micronutrientes Zn, Cu e $B$, foi verificada uma redução da MSSR, em relação ao tratamento $\mathrm{C} 1$, porém, na MSPA, quando se omitiu $\mathrm{Zn}$ e $\mathrm{Cu}$, não houve diferença estatística em relação ao C1 (Figura 1).

No tratamento C1-Zn a MSSR e a MSPA corresponderam a 139,83\% e a $38,4 \%$, respectivamente daquelas obtidas no tratamento C1 (Figura 2). Segundo Marschner (1995), tanto a toxidade como a falta de zinco pode afetar o crescimento e o metabolismo normal das espécies vegetais. Malavolta (1989), afirma que o zinco é indispensável para a produção de clorofila. De acordo com Dechen et al., (1991), a deficiência de $\mathrm{Zn}$ pode se associar ao excesso de $\mathrm{P}, \mathrm{Mn}$ e F no solo, e de $\mathrm{P}$ e Cu na planta.

O cobre é importante para a ativação de enzimas, síntese de proteínas, metabolismo de carboidratos e fixação de $\mathrm{N}_{2}$ (Malavolta, 1989). No presente estudo a produção de MSSR e a MSPA corresponderam, respectivamente, a $122,76 \%$ e 48,13\% daquelas obtidas no tratamento C1 (Figura 2).

Diferentemente da omissão do Zn e do $\mathrm{Cu}$, a omissão de $\mathrm{B}$ limitou tanto a MSSR quanto a MSPA. No tratamento C1-B a MSSR e PSPA corresponderam a $38,6 \%$ e a $23,5 \%$ daquelas obtidas no tratamento C1 (Figura 2). A deficiência de boro prejudica o transporte e a ação dos reguladores de crescimento, além de provocar distúrbios no desenvolvimento da planta, em razão do aumento do nível do ácido indol acético, redução da síntese de proteínas, dificuldade de formação da parede celular e no transporte de produtos da fotossíntese, propiciando o acúmulo de compostos fenólicos no vegetal (Melo \& Lemos, 1991). Segundo Malavolta (1989) a ausência de boro na planta, pode provocar a morte do meristema apical, também chamada de seca dos ponteiros. De acordo com Primavesi (1990), a deficiência de boro é a que mais drasticamente reduz o volume radicular, tal fato observado neste experimento, onde se verificou que o tratamento com a omissão de B, a produção de MSSR foi a menor, correspondendo a 23,59\% em comparação ao tratamento C1 (Figura 2).

Além da redução na produção de biomassa, na ausência de macronutrientes e micronutrientes, não foram verificados sintomas visíveis de deficiência nutricional nas plantas do mangarito. A ordem decrescente dos tratamentos que mais afetaram a produção de matéria seca da parte aérea foi: Calagem $>-\mathrm{P}>-\mathrm{S}>$ testemunha $>-\mathrm{B}>$ -Calagem+Ca+Mg $>-\mathrm{N}>-\mathrm{K}$. A omissão de $\mathrm{Ca}$, Mg, Zn e Cu não afetaram negativamente a produção de matéria seca da parte aérea. A ordem decrescente dos tratamentos que mais afetaram a produção de matéria seca do sistema radicular foi: $-\mathrm{B}>-\mathrm{Mg}>-$ Calagem $+\mathrm{Ca}+\mathrm{Mg}>-\mathrm{K}>-\mathrm{N}>-\mathrm{Zn}>-$ Calagem $>-\mathrm{S}>-\mathrm{Cu}>-\mathrm{Ca}>-\mathrm{Cu}>-\mathrm{Ca}$ $>$ testemunha $>-\mathrm{P}$.

\section{REFERÊNCIAS}

AGUIAR APA. 1998. Manejo da fertilidade do solo sob pastagem, calagem e adubação. Guaíba: Agropecuária. 120p. 
ANJOS JT; PUNDEK M; TASSINARI G; GRIMM SS. 1981. Efeito da calagem e da adubação fosfatada sobre algumas propriedades químicas de um Cambissolo Húmico distrófico cultivado com milho. Revista Brasileira de Ciência de Solo 5: 50-54.

ARAÚJO RC. 2001. Produção, qualidade dos frutos e teores foliares de nutrientes no maracujazeiro amarelo em resposta à nutrição potássica. Viçosa: Universidade Federal de Viçosa. 103p. (Tese doutorado).

CARNEIRO JGA. 1995. Produção e controle de qualidade de mudas florestais. Curitiba: FUPEF, 451p.

CHAMINADE R. 1972. Recherches sur la fertilité et la fertilisation des sols en régions tropicales. Agronomie Tropicale, Montpellier 27: $891-$ 904.

COURSEY DG. 1968. The edible aroids. World Crops, London, 20: 3-8.

DECHEN AR; HAAG HP; CARMELO QAC. 1991. Funções de micronutrientes nas plantas. In: FERREIRA ME; CRUZ MCP. (Ed.). Micronutrientes na agricultura. Piracicaba: POTAFOS/CNPq. p. 65-78.

EMPRESA BRASILEIRA DE PESQUISA AGROPECUÁRIA. 1997. Serviço Nacional de Levantamento e Conservação de Solo. Manual de métodos de análise de solo. Rio de Janeiro: EMBRAPA, SNLCS. 212p.

ENY BAC. 1967. Effect of spacing, sett size, ridging and mulching on the development and yield of cocoyam (Xanthosoma sagittifolium Schott). Tropical Agriculture 44: 53-60.

ERNANI PR; FIGUEIREDO ORA; BECEGATO V; ALMEIDA JA. 1996. Decréscimo da retenção de fósforo no solo pelo aumento do pH. Revista Brasileira de Ciência de Solo 20: 159-162.

HARIDASAN M. 2000. Nutrição mineral de plantas nativas do cerrado. Revista Brasileira de Fisiologia Vegetal 12: 54-64.
FERNANDES LA; FAQUIN V; FURTINI NETO AE; CURI N. 2000. Frações de fósforo e atividade de fosfatase ácida em plantas de feijoeiro cultivadas em solos de várzea. Revista Brasileira de Ciência de Solo 24: 561-571.

GODDARD RE; HOLLIS CA. 1984. The genetic basics of forest tree nutrition. In: BOWEN GD; NAMBIER EKS. (Ed.) Nutrition of plantation forest. London: Academic Press. p.237-258.

GOMES JM; PAIVA HN. 2004. Viveiros Florestais: propagação sexuada. Viçosa: UFV. $116 \mathrm{p}$.

GOMES MAF. 2000. Nutrientes vegetais no meio ambiente: ciclos bioquímicos, fertilizantes e corretivos. Jaguariúna: Embrapa Meio Ambiente. 50p.

GRAHAM RD. 1984. Breeding for nutritional characteristics in cereals. In: TINKER PB; LAUCHLI A. (Ed.) Advances in Plant Nutrition. New York: Praeger. p.57-102.

JORDAN FL. 1979. Preliminary work whit tanier (Xanthosoma spp.) in Puerto Rico. Universidad Puerto Rico, 63: 469-473.

JUNQUEIRA AH; LUENGO RFA. 2000. Mercados diferenciados de hortaliças. Horticultura Brasileira 18: 98-99.

KLEIN VL; BRANDÃO M; LACA-BUENDIA JP. 1989. Cyclanthera pedata var. edulis (Naud.) Cogn. Uma Cucurbitaceae pouco conhecida na alimentação humana. Acta Botanica Brasilica 1: 8-13.

LOPES AS. 1983. Solos sob cerrado: características, propriedades e manejo. Piracicaba: Potafos. 162p.

MALAVOLTA E. 1980. Elementos de nutrição mineral de plantas. São Paulo: Agronômica Ceres. 251p.

MALAVOLTA E. 1986. Micronutrientes na adubação. Paulínia: Nutriplant. 70p.

MALAVOLTA E. 1989. ABC da adubação. São Paulo: Agronômica Ceres Ltda. 292p.
MALAVOLTA E; VITTI GC; OLIVEIRA SA. 1997. Avaliação do estado nutricional das plantas: princípios e aplicações. Piracicaba: Associação Brasileira para Pesquisa da Potassa e do Fosfato. 210p.

MARSCHNER H. 1995. Mineral nutrition of higher plants. London: Academic Press. 889p.

MELO JW; LEMOS EGM. 1991. Análise bioquímica de plantas. In: FERREIRA ME; CRUZ MCP. (Ed.). Micronutrientes na agricultura. Piracicaba: POTAFOS/CNPq. p. 309-332.

MENGEL K; KIRKBY EA. 1987. Principles of plant nutrition. Bern, International Potash Institute. 687p.

PENA RS. 1970. The edible aroids in the Asian Pacific Area. In: INTERNATIONAL SIMPOSIUM ON TROPICAL ROOT AND TUBER CROPS, 2., Honolulu, 1970. Proceedings. College of Hawaii of Tropical Agriculture, University of Hawaii 1: 136-140.

RAIJ BV. 1990. Potássio: Necessidade e uso na agricultura moderna. Piracicaba: POTAFOS. 45p.

SCHELLER E. 1998. Fundamentos científicos da nutrição vegetal na agricultura ecológica. 78p.

VASCONCELOS EFC. 1972. Estudo sobre espaçamento e tipos de rizomas na propagação e produção do mangará Xanthosoma mafaffa Schott. Piracicaba: ESALQ-USP. 139p. (Tese doutorado).

TAIZ L; ZEIGER E. 2004. Fisiologia Vegetal. Porto Alegre: Artimed ed. 19p. 